\title{
Cessation of long-acting $\beta 2$-agonist in children with persistent asthma on inhaled corticosteroids
}

\section{To the Editor:}

International guidelines recommend adding long-acting $\beta 2$-agonists (LABA) to inhaled corticosteroids (ICS) as a step 3 or 4 strategy in children in whom ICS, with or without adjunct therapy, is ineffective in adequately controlling asthma [1-4]. Contrary to adults, the beneficial effects of ICS/LABA in children are limited to improving lung function and short-acting $\beta 2$-agonist use (SABA), with no significant reduction in symptoms compared with ICS alone [5]; moreover, a nonsignificant trend towards more exacerbations requiring oral corticosteroids and/or hospital admissions raised concerns [5]. Asthma-related intubations and deaths linked to LABA [6] have led the US Food and Drug Administration to issue label changes recommending that LABA is discontinued once asthma control has been achieved [7].

Two systematic reviews concluded that, in adults with adequate asthma control on ICS/LABA, stepping-off LABA to the same ICS dose led to: a three-fold increase in patient withdrawals due to poor control; a slight decrease in asthma control, quality of life and symptom-free days; and a trend towards more oral corticosteroids use $[8,9]$. With no published paediatric trials exploring the outcome of LABA cessation strategies [9], there is little evidence to guide physicians regarding the expected duration of ICS/LABA therapy, optimal cessation strategies and predictors of successful LABA cessation in children $[4,7,10]$.

We conducted a retrospective cohort study of children aged 1-17 years, with a diagnosis of asthma [11], on maintenance ICS, and who were initiated on ICS/LABA (the cohort entry date) in the Asthma Clinic of the Sainte-Justine University Hospital Centre. Parents provided consent to enrol in the Paediatric Asthma Database and Biobank (including detailed patient and treatment information) with access to all administrative hospital databases via the "Banque de données cliniques, administratives et de recherche chez l'enfant (B-CARE)" and to the "reMed database" providing drug claim data from pharmacies across the province of Quebec for privately insured and a proportion of publically insured patients. The cohort end date was 6 months after LABA cessation or July 1, 2015, whichever occurred first. The protocol was approved by the Institutional Review Board (CHU Sainte-Justine, Montreal, Canada). The main outcome was the proportion of successful LABA cessation, defined as a 6-month period with physician-reported good/excellent asthma control, no rescue oral corticosteroids and no LABA re-initiation. A sensitivity analysis was performed in the subgroup with drug claims data, using less than four SABA doses per week as a marker of control.

Between 2011 and 2014, 106 children met the eligibility criteria, with drug claim data available in 63 patients. Participants were predominantly male school-aged children $(73.6 \%$ aged $\geqslant 4$ years) with persistent asthma, who had suboptimal asthma control and mild airway obstruction on a medium ICS dose. Children were overwhelmingly prescribed a combination of fluticasone/salmeterol with a medium ICS dose as a step 3 strategy. During the ICS/LABA treatment period, half of the patients maintained unchanged ICS and LABA doses. Nearly $60 \%$ of patients that attempted down-titration reduced their ICS/ LABA frequency from twice to once daily, with no subsequent increase in dosage during ICS/LABA therapy in most patients. When unsuccessful, however, LABA cessation was seldom attempted.

After a marked response in terms of lung function and asthma control, LABA was discontinued in 53 $(50 \%)$ of the whole cohort. The median (interquartile range) duration on ICS/LABA was 1.5 (1.12.1) years. Similarly, 40 (64\%) of those with drug claim data discontinued LABA after $1.6(1.3-2.1)$ years. Children switched to the same (40\%), a lower (36\%) or a higher (6\%) ICS dose; 19\% stepped off ICS/ LABA to either no daily controller $(11 \%)$ or montelukast $(8 \%)$. The primary outcome, that is, the proportion of children with successful cessation was $83 \%$ of 53 children in the whole cohort and $55 \%$ of 40 children with drug claims data (table 1).

No demographic or treatment characteristics were significantly associated with successful LABA cessation in the whole cohort, although the duration of good or excellent control prior to cessation approached statistical significance (OR 1.14, 95\% CI 1.00-1.30). In the subgroup with drug claim data (with a higher failure rate), a longer duration of ICS/LABA therapy (OR 1.26, 95\% CI 0.07-1.48) was positively associated with successful LABA cessation, whereas ICS down-titration prior to LABA cessation (OR 0.05, 95\% CI $0.00-0.84$ ) and montelukast monotherapy at cessation (OR $0.00,95 \%$ CI $0.00-0.77$ ) were negatively associated with successful LABA cessation. 
TABLE 1 Successful cessation in children who stopped inhaled corticosteroids (ICS)/ long-acting $\beta 2$-agonists (LABA) during the observation period

All participants

Participants with

drug claims data

\section{Subjects \\ Successful cessation ${ }^{\#}$ \\ Unsuccessful cessation"}

Poor/unsatisfactory physician global assessment of control

Use of rescue oral corticosteroids at least once

Recurrence of ICS/LABA use

Average use of short-acting $\beta 2$-agonist $\geqslant 4$ doses per week
53

$44(83.0)$

$6(11.3)$

$3(5.7)$

$7(13.2)$

n/a
40

$22(55.0)$

$\mathrm{n} / \mathrm{a}$

$3(7.5)$

$7(17.5)$

$12(30.0)$

Data are presented as $\mathrm{n}$ or $\mathrm{n}(\%) . \mathrm{n} / \mathrm{a}$ : not available. ${ }^{\#}$ : For the group consisting of all participants, successful cessation was defined as: 1) good or excellent asthma control based on physician global assessment; 2) no rescue oral corticosteroids; and 3) no recurrence of ICS/LABA use. For participants with drug claims data, physician global assessment of asthma control was replaced by less than four weekly doses of rescue short-acting 32 -agonist (SABA) based on SABA drug servings during the 6-month period following ICS/LABA cessation, a criteria recognised as a more sensitive measure of asthma control than physician assessment and exacerbations [12]. ": Patients could meet more than one criteria for unsuccessful cessation, recorded in the electronic medical records (Paediatric Asthma Database and Biobank) and the hospital administrative database (Banque de données cliniques, administratives et de recherche chez l'enfant (B-CARE)), including acute care visits, for all participants or documented by the reMED database for those with drug claims data.

In this paediatric cohort study, the median duration of 18 months on ICS/LABA treatment appears robust as confirmed by the sensitivity analysis. With no description of treatment duration prior to cessation in adult cessation trials, unless imposed by the study design $[8,9]$, ours appear as the first report of ICS/LABA duration in the real-life setting.

Using 6 months to define successful cessation, $83 \%$ (or $55 \%$ in the drug claims analysis) successfully discontinued LABA, which is consistent with the only identified observational paediatric study in which $63 \%$ of children maintained asthma control after 2 months of LABA cessation [13]. Potential explanations for the higher success rate in our study compared with adult trials [8] include less airway obstruction and remodelling, shorter disease duration, higher ICS dose to which LABA was added and stepped off to, longer duration of asthma control prior to LABA cessation, and lower benefit of LABA in children [5].

As a predictor of successful LABA cessation, the duration of asthma control prior to LABA cessation did not meet statistical significance, perhaps because of insufficient power, whereas three important determinants of success were identified in the sensitivity analysis. We hypothesise that duration of ICS/ LABA therapy and avoidance of ICS step-down probably increased the probability of overcoming airway inflammation prior to LABA cessation. Avoidance of montelukast monotherapy, a treatment inferior to ICS monotherapy, as a predictor of success underlines the importance of maintaining sufficient anti-inflammatory therapy after LABA cessation [14]; yet, it is unclear why the few who stopped all daily controllers were not at an increased risk of failure. No other factors, including age group ( $<4$ years), ICS dose after discontinuation and adherence, were significantly associated with successful LABA cessation.

Until recently, research efforts and guidelines have been focused on reducing the ICS, and not the LABA, dose $[1,10,15]$. Throughout ICS/LABA therapy, half of our patients maintained a stable dose of ICS and LABA. To our knowledge, there are no trials exploring different ICS and/or LABA down-titration strategies in head-to-head comparisons, a gap in knowledge worth exploring as ICS down-titration was associated with more LABA cessation failures.

Although it represents an off-label use of LABA, we included children $<4$ years of age, an age group in which ICS/LABA is used in a tertiary care centres for difficult-to-treat children despite the absence of randomised trials of adjunct therapy: age was not a significant determinant of success. Successful cessation was defined by rigorous criteria, yet mild symptoms or quality of life impairment without SABA use may have been missed, although the justification to restart LABA in such cases may be debated. The study was conducted in the Canadian province of Quebec with free access to medical care and subsidised asthma medication for all children and in a single tertiary care paediatric asthma clinic staffed by paediatric specialists: we advise caution in generalising our findings to other settings until replication.

Half of patients discontinued LABA by 18 months of therapy and most retained satisfactory asthma control for at least 6 months. Maintaining a sufficient ICS dose prior to, and after, LABA cessation appeared as marker of successful discontinuation, although this must be confirmed in replication studies. 
We underline the paucity of trials to guide LABA cessation in children. We recommend that such trials begin at LABA initiation, ensure a sufficient duration of asthma control prior to stepping off to ICS monotherapy, and compare different ICS or LABA down-titration strategies prior to cessation.

@ERSpublications

Most asthmatic children can be successfully weaned off long-acting $\beta 2$-agonists to inhaled corticosteroid monotherapy http://ow.ly/10oTtI

Francine M. Ducharme ${ }^{1,2,3}$, Roxanne Gagnon ${ }^{2}$, Brigitte Bénard ${ }^{2}$, Sze Man Tse ${ }^{1,2}$ and Robert Thivierge ${ }^{1}$

${ }^{1}$ Dept of Pediatrics, University of Montreal, Montreal, QC, Canada. ${ }^{2}$ Clinical Research and Knowledge Translation Unit on Childhood Asthma, Research Centre, CHU Sainte-Justine, Montreal, QC, Canada. ${ }^{3}$ Dept of Social and Preventive Medicine, University of Montreal, Montreal, QC, Canada.

Correspondence: Francine M. Ducharme, CHU Sainte-Justine, 3175 Côte Ste-Catherine, Room 7939, Montreal, Quebec, H3T 1C5, Canada. E-mail: francine.m.ducharme@umontreal.ca

Received: Nov 202015 | Accepted after revision: March 302016 | First published online: May 262016

Support statement: The investigator-initiated study was funded by a grant from Merck Canada in the Merck Investigator Studies Program (grant number: 50925), but the sponsor had no input in the study design, conduct, analysis or writing of the manuscript. We acknowledge the support of the Fonds de la Recherche en Santé du Québec for the infrastructure support provided to the Research Institute of the Sainte-Justine University Health Centre. Funding information for this article has been deposited with FundRef.

Conflict of interest: Disclosures can be found alongside this article at erj.ersjournals.com

Acknowledgements: We are indebted to Pierre Gaudreault (University of Montreal, Montreal, Canada) and Caroline Chartrand (CHU Sainte-Justine, Montreal, Canada) who assisted in the diligent data collection during their patients' medical visits and thank the parents of the children enrolled in this study.

\section{References}

1 Global Initiative for Asthma (GINA). 2015 GINA report, Global strategy for asthma management and prevention. www.ginasthma.org/ Date last accessed: November 15, 2015.

2 British Thoracic Society; Scottish Intercollegiate Guidelines. British guideline on the management of asthma. Thorax 2014; 69: Suppl., 1-192.

3 Lougheed MD, Lemiere C, Ducharme FM, et al. Canadian Thoracic Society 2012 guideline update: diagnosis and management of asthma in preschoolers, children and adults. Can Respir J 2012; 19: 127-164.

$4 \quad$ National Heart Lung and Blood Institute. Expert panel report 3. Guidelines for the diagnosis and management of asthma. Bethesda, National Institutes of Health, 2007.

5 Chauhan BF, Chartrand C, Ni Chroinin M, et al. Addition of long-acting beta-agonists to inhaled corticosteroids for chronic asthma in children. Cochrane Database Syst Rev 2015; 11: CD007949.

6 Salpeter SR, Wall AJ, Buckley NS. Long-acting beta-agonists with and without inhaled corticosteroids and catastrophic asthma events. Am J Med 2010; 123: 322-328.

7 Chowdhury BA, Dal Pan G. The FDA and safe use of long-acting beta-agonists in the treatment of asthma. N Engl J Med 2010; 362: 1169-1171.

8 Brozek JL, Kraft M, Krishnan JA, et al. Long-acting beta2-agonist step-off in patients with controlled asthma. Arch Intern Med 2012; 172: 1365-1375.

9 Kew KM, Beggs S, Ahmad S. Stopping long-acting beta2-agonists (LABA) for children with asthma well controlled on LABA and inhaled corticosteroids. Cochrane Database Syst Rev 2015; 5: CD011316.

10 Reddel HK, Gibson PG, Peters MJ, et al. Down-titration from high-dose combination therapy in asthma: removal of long-acting $\beta 2$-agonist. Respir Med 2010; 104: 1110-1120.

11 Ducharme F, Dell SD, Radhakrishnan D, et al. Diagnosis and management of asthma in preschoolers: a Canadian Thoracic Society and Canadian Paediatric Society position paper. Can Respir J 2015; 22: 135-143.

12 Ducharme FM, Noya FJ, Allen-Ramey FC, et al. Clinical effectiveness of inhaled corticosteroids versus montelukast in children with asthma: prescription patterns and patient adherence as key factors. Curr Med Res Opin 2012; 28: 111-119.

13 O'Hagan AR, Morton R, Eid N. Loss of asthma control in pediatric patients after discontinuation of long-acting beta-agonists. Pulm Med 2012; 2012: 894063.

14 Chauhan BF, Ducharme FM. Anti-leukotriene agents compared to inhaled corticosteroids in the management of recurrent and/or chronic asthma in adults and children. Cochrane Database Syst Rev 2012; 5: CD002314.

15 Bateman ED, Jacques L, Goldfrad C, et al. Asthma control can be maintained when fluticasone propionate/ salmeterol in a single inhaler is stepped down. J Allergy Clin Immunol 2006; 117: 563-570. 